Technological University Dublin

DÜBLIN

ARROW@TU Dublin

Articles

Centre for Industrial and Engineering Optics

2017

\title{
LTL Type Nanozeolites Utilized in Surface Photonics Structures for Environmental Sensors
}

\author{
Sabad-e- Gul \\ Technological University Dublin, sabade.gul@mydit.ie \\ Dervil Cody \\ Technological University of Dublin, dervil.cody@tudublin.ie \\ Suzanne Martin \\ Technological University of Dublin, suzanne.martin@tudublin.ie
}

See next page for additional authors

Follow this and additional works at: https://arrow.tudublin.ie/cieoart

Part of the Earth Sciences Commons

\section{Recommended Citation}

Cody, D., Gul, S.-E. \& Kharchenko, A. (2017). LTL Type Nanozeolites Utilized in Surface Photonics Structures for Environmental Sensors. Microporous and Mesoporous Materials, vol. 261, pp. 268-274. doi:10.1016/j.micromeso.2017.11.019

This Article is brought to you for free and open access by the Centre for Industrial and Engineering Optics at ARROW@TU Dublin. It has been accepted for inclusion in Articles by an authorized administrator of ARROW@TU Dublin. For more information, please contact arrow.admin@tudublin.ie, aisling.coyne@tudublin.ie, gerard.connolly@tudublin.ie.

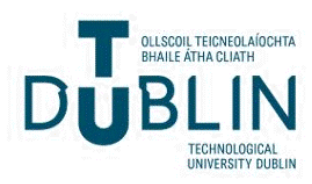




\section{Authors}

Sabad-e- Gul, Dervil Cody, Suzanne Martin, John Cassidy, Izabela Naydenova, Anastasia Kharchenko, and Svetlana Mintovab 


\title{
LTL type nanozeolites utilized in surface photonics structures for environmental sensors
}

\author{
Sabad-e Gul ${ }^{\mathrm{a}}$, Dervil Cody ${ }^{\mathrm{a}}$, Anastasia Kharchenko ${ }^{\mathrm{b}}$, Suzanne Martin ${ }^{\mathrm{a}}$, Svetlana Mintova ${ }^{\mathrm{b}}$, \\ John Cassidy ${ }^{\mathrm{c}}$, Izabela Naydenova ${ }^{\mathrm{a}, *}$

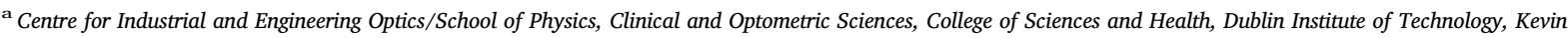 \\ Street, Dublin 8, Ireland \\ ${ }^{\mathrm{b}}$ Laboratoire Catalyse \& Spectrochimie, ENSICAEN, Université de Caen Basse-Normandie, CNRS, 6, Boulevard du Maréchal Juin, 14050 Caen Cedex, France \\ ${ }^{\mathrm{c}}$ School of Chemical and Pharmaceutical Sciences, College of Sciences and Health, Dublin Institute of Technology, Kevin Street, Dublin 8, Ireland
}

\section{A R T I C L E I N F O}

\section{Keywords:}

Zeolite nanoparticles

Nanosized zeolite based sensors

Water monitoring

Copper ions

Holography

Modified surface structures

Sensors

\begin{abstract}
A B S T R A C T
Environmental monitoring has been continuously increasing due to an emerging global emphasis on the importance of sustainable development. Improving water quality monitoring and control is a challenge that calls for innovative solutions. Current methods of water monitoring are costly and analytical techniques suitable for field use are limited. There is a need for accurate, long-term monitoring of environmental contaminants using sensors that can be operated on site. The aim of our research is to theoretically model, fabricate and characterise holographic sensors for water quality monitoring that are simple to operate, capable of sensing copper present in fresh water and have relatively low cost. The sensors are created by holographic recording of surface relief gratings (SRG) in a self-processing photopolymer material. Interrogation of these structures by light allows indirect measurements of ion concentration in real time. The SRG structures are modified by coating with porous LTL-nanoparticles (nanosized zeolites) which selectively adsorb copper ions. The suitability of the sensors for detection of copper (II) present in water at concentration levels 1-4 $\mathrm{mM}$ is reported. The current detection limit of the sensor is $63 \mathrm{ppm}$.
\end{abstract}

\section{Introduction}

Environmental monitoring is essential to protect the environment from toxic contaminants. Water is progressively becoming more polluted in our environment due to discharge of large amounts of metal ion-contaminated wastewater by industry directly into the water supply. These metal ion contaminants need to be removed before recycling or discharging directly into the surface water in order to prevent toxic conditions and serious health problems as a result of exposure [1]. Interestingly, small amounts of metal elements are common in our environment and diet, which are necessary for good health. However, in excessive amounts heavy metals are toxic; short term exposure results in damaged or reduced central nervous functions, lower energy levels, damaging lungs, kidneys, liver and other vital organs. Long term exposure may result in slowly progressing physical, muscular and neurological degenerative processes that mimic cancer $[1,2]$. For some heavy metals, toxic levels can be just above the background concentration naturally found in nature. The maximum contamination levels of some heavy metal ions for safe drinking water are nickel: $2 \mathrm{mg} /$ $\mathrm{dm}^{3}$, copper: $1.3 \mathrm{mg} / \mathrm{dm}^{3}$, chromium: $0.10 \mathrm{mg} / \mathrm{dm}^{3}$, manganese: $0.05 \mathrm{mg} / \mathrm{dm}^{3}$, iron: $0.5 \mathrm{mg} / \mathrm{dm}^{3}$, arsenic: $10 \mu \mathrm{g} / \mathrm{dm}^{3}$ and mercury: $2 \mu \mathrm{g} / \mathrm{dm}^{3}$ [2]. Therefore, it is important to be aware of heavy metal toxicity and to take protective measures against excessive exposures.

The majority of existing monitoring methods are costly and time consuming. Sampling and analytical techniques can be insufficient and difficult to implement [3]. Chemical sensors have generated a great interest in the determination of various pollutants and can play a substantial role in environmental monitoring [2]. These devices offer remote detection, miniaturization enabling at-site measurements and minimal waste production, which contributes to a better quality of life.

Holography allows fabrication of disposable photonic sensors that are lightweight [4]. This is useful for miniaturization and multiplexing purposes. Some potential applications of holographic sensors are in veterinary testing, medical assays and environmental monitoring.

One of the possible solutions in the development of effective environmental sensors could be the application of zeolite crystalline nanoparticles which can be applied in the design and fabrication of photonic structures. Currently the main application is in catalysis and

\footnotetext{
* Corresponding author.

E-mail address: izabela.naydenova@dit.ie (I. Naydenova).
} 
membrane design, however, zeolites have several unique features which make them suited for the fabrication of water pollutant sensors. Zeolites are characterised by a highly porous structure with a pore size similar to the size of many industrially important molecules, and the trapping of a variety of chemicals in zeolites is well documented [5-8]. Due to the large variety of zeolite nanoparticles structure and chemical composition, the flexibility in their synthesis and the possibility to functionalise them, these nanoparticles are ideal candidates for incorporation in sensors. This paper describes sensors based on surface relief grating (SRG) structures created by holographic recording in photopolymers. These holographic structures constitute a flexible platform for fabrication of sensor devices since they can be modified with the help of different materials and thus make them selective to a specific analyte. An example of detecting pollutants such as copper ions in water is presented.

Holographic sensors have been attracting significant attention in recent years due to their ability to be interrogated optically, providing either visual or electronic output $[4,6]$. They can be multiplexed, miniaturised, their response experiences little interference from external electromagnetic fields, they can be designed to perform remote sensing. Similar to other sensing technologies, the main challenges in holographic sensors are related to providing better selectivity, sensitivity, fast response time, good repeatability and reversibility. Until recently holographic sensors have been predominantly based on volume holograms, reflection or transmission $[4,9]$. The change in the hologram properties is observed due to either dimensional changes of the hologram or change of the refractive index/refractive index modulation under exposure to the analyte. Using volume holograms intrinsically offers higher sensitivity, due to the higher theoretically achievable diffraction efficiency and due to the fact that these devices are generally thicker and thus even a minute change in the refractive index/refractive index modulation or dimension of the material can lead to a significant change in the diffractive properties of the structure. On the other hand, the target molecules have to travel larger distances in order to penetrate the structure, thus these devices are generally expected to be slower in their response. In addition variation of their selectivity is not a straight forward process since it requires both variation of the chemical composition of the polymer layer to include a different functionalising material and protection of the ability of the photosensitive layer to record a hologram. Some of these challenges and particularly the last one can be addressed by utilisation of SRG photonic structures instead of volume holograms.

\section{Surface relief structures and their application in sensing}

The primary advantage of surface relief grating structures for sensing is that the target analyte is not required to permeate fully into the structure as is the case for volume grating holographic sensors; interaction of the target analyte with the surface structure will result in a measurable change in the sensor output. This will potentially enhance the device sensitivity and facilitate faster response times. Full reversibility and reusability of the sensor, if desirable, may also be easier to achieve. A disadvantage of surface relief structure sensors is that the sensitivity of the device is governed by the aspect ratio of the surface relief grating i.e. the maximum achievable surface relief amplitude as a function of grating period. Sensors based on surface relief gratings with higher aspect ratios will produce a greater sensor response as a function of analyte concentration. However, the ability to achieve large surface relief amplitudes at high spatial frequency is governed by the properties of the holographic recording medium. The use of SRG [10-12], and recently more complex diffractive optical elements [13] as sensors have been previously successfully implemented and reported. Analyte induced change of the surface relief amplitude and/or refractive index modulation have been utilized as detection mechanisms. Surface relief structures feature across a range of optical sensor platforms including surface plasmon resonance-based sensors for detection of chemical and biological molecules [14] and optical fibre Bragg grating-based sensors for volatile organic compounds (VOC) detection [4,15] and temperature monitoring [16] have been reported. The inscription of the surface of molecularly-imprinted polymers via interference photolithography has facilitated the production of testosterone sensors [17]. Aztec holograms which are essentially a combination of the surface structure and Bragg reflector geometries, were originally proposed in Ref. [18] and have recently been functionalized for relative humidity sensing via coating with hydrophilic materials such as polyvinyl alcohol and glycerol [19].

Here, a sensor based on the structure of a surface relief grating inscribed holographically in a photopolymer layer is proposed. The period, $\Lambda$, and surface relief amplitude, $d$, of the grating are controlled via manipulation of the holographic recording conditions. Depending on these parameters, the surface relief grating may be defined as either thin or thick, in accordance with the Klein-Cook Q parameter [20]:

$Q=\frac{2 \pi \lambda_{r} d}{n \Lambda^{2}}$

where $n$ is the recording medium refractive index and $\lambda_{r}$ is the reconstruction wavelength. $\mathrm{Q}$ values larger than 10 correspond to thick gratings which may be described using Kogelnik's Coupled Wave Theory [21], whereas gratings with a $Q$ value of less than 1 are considered in the thin regime, and may be described using Raman-Nath Theory [22]. Surface relief gratings fabricated in photopolymer media are typically in the thin regime, as the surface relief amplitudes required to be classified as thick are very difficult to achieve at the necessary grating spatial frequencies.

The output of the surface relief grating-based sensor as a function of analyte concentration is measured via change in grating diffraction efficiency, $\eta$. For thin phase gratings in the Raman-Nath regime, $\eta$ is defined as:

$\eta=J_{m}^{2}\left(\frac{\pi \Delta n d}{\lambda_{r}}\right)$

Where $J_{m}$ is the Bessel function of the order $m$. The incident beam is diffracted into a number of orders, with the diffracted amplitude in the $m$ th order proportional to the value of the Bessel function. $\Delta n$ is the refractive index modulation of the grating, which is the difference between the maximum and minimum refractive index in the structure under consideration.

\subsection{Modified surface relief grating (SRG)}

In order to facilitate absorption of the target analyte molecules and enhance sensor function, the surface relief grating is modified through coating with zeolite nanoparticles. Here, the SRG acts as a support structure for the zeolites, and $\Delta n$ is defined as the difference between the refractive index of the support structure (i.e. photopolymer) and the zeolite nanoparticles. On exposure to an analyte, the analyte molecules exchange into the zeolite nanoparticles, resulting in a change in $\Delta n$, which in turn produces a measurable change in the $\eta$ of the sensor as a function of analyte concentration (Fig. 1).

\section{Experimental}

\subsection{Materials}

\subsubsection{Photopolymer layers}

The acrylamide based photopolymer was prepared as described in Table 1 . The components were well mixed in water by using a magnetic stirrer. All the materials used in this work were of analytical grade purchased from Sigma Aldrich without further purification.

All the samples were prepared under ambient conditions (temperature $16-22{ }^{\circ} \mathrm{C}$; relative humidity (RH), 40-60\%) as follows. PVA (MW 9000-10,000) was dissolved in distilled water while heated at 
a)

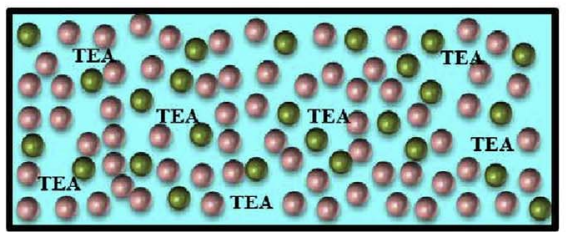

c)

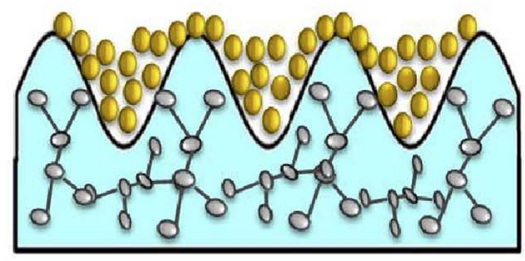

b)

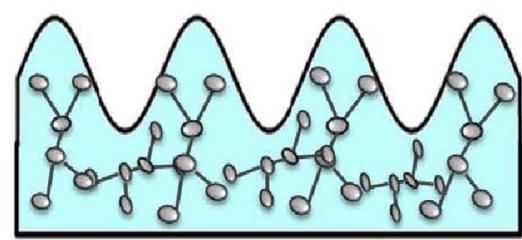

d)

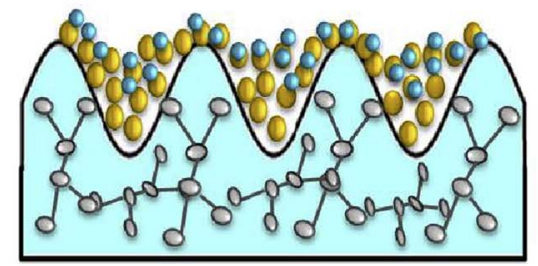

Fig. 1. Distribution of constituents of photopolymerisable nanocomposites: a) acrylamide (AA) $\boldsymbol{\Theta}$, bisacrylamide (BAA) $\odot$ monomer molecules and initiator Triethanolamine (TEA), b) after polymerization, c) 0 after coating with LTL-zeolite nanoparticles and d) exposure to analyte.
Table 1

The amount of component's added in the photopolymer solution.

\begin{tabular}{lll}
\hline Components & Amount (g) & $\begin{array}{l}\text { Amount }(\% \mathrm{w} / \mathrm{w}) \text { in dry } \\
\text { layer }\end{array}$ \\
\hline Acrylamide (AA) & 1.0 & 24.5 \\
N,N'Methylenebisacrylamide (BAA) & 0.2 & 4.9 \\
Polyvinyl alcohol (10\% wt/wt) (PVA) & 1.75 & 43.0 \\
Triethanolamine (TEA) & 1.12 & 27.5 \\
Erythrosine B dye (0.001\% wt/wt) & 0.0044 & 0.1 \\
\hline
\end{tabular}

$70{ }^{\circ} \mathrm{C}$ to yield a $10 \mathrm{wt} \%$ PVA aqueous solution. Crystals of Erythrosine B (ErB) were dissolved in water at room temperature to obtain dye stock solution with the desired dye concentration $(0.001 \mathrm{wt} \%)$. The triethanolamine (TEA) was added into the PVA solution. Finally, acrylamide (AA) and $\mathrm{N}, \mathrm{N}^{\prime}$ Methylenebisacrylamide) BAA were added as the monomers, along with the ErB solution. After mixing for $1 \mathrm{~h}$ all components were fully dissolved and the photopolymer solution had a total volume of approximately $23 \mathrm{ml} .30 \mu \mathrm{l}$ volume of photopolymer solution was spread evenly on a glass slide of dimensions $7.6 \mathrm{~cm} \times 2.6 \mathrm{~cm}$. The coated glass slide was placed on a flat surface and allowed to dry in dark for typically 5-6 h. The thickness of the dry sample was $30 \pm 3 \mu \mathrm{m}$.

\subsubsection{LTL nanoparticles synthesis}

Zeolite nanocrystals with LTL-type structure were prepared from clear precursor solutions, free of organic template, with the following molar composition: 5 K2O: 10 SiO2: 0.5 A12O3: 200 H2O. The asprepared precursor solutions were aged at room temperature for $24 \mathrm{~h}$ prior to hydrothermal treatment at $170{ }^{\circ} \mathrm{C}$ for $18 \mathrm{~h}$. After crystallization, the nanosized crystals were recovered by multistep centrifugation (20000 rpm, $40 \mathrm{~min}$ ) and washed with doubly distilled water. The LTL nanocrystals were stabilized in water at $\mathrm{pH}=8$ and with concentration of solid particles of about $1.5 \mathrm{wt} \%$ [23].

\subsection{Methods}

\subsubsection{Recording of the surface relief gratings (SRG)}

A two-beam holographic optical setup used to record the holographic gratings is shown in Fig. 2. The angle between the beams was set as $9.16^{\circ}$ in order to obtain an interference pattern with spatial frequency of 300 lines $/ \mathrm{mm} \pm 10$ lines $/ \mathrm{mm}$. An Nd: YVO4 laser $(532 \mathrm{~nm})$ was used to record the transmission holographic gratings. The recording intensity was $10 \mathrm{mWcm}^{-2}$ and the exposure time was $100 \mathrm{~s}$.
The recording beams were $\mathrm{S}$ polarized in order to obtain optimum recording conditions. The recording conditions were previously optimised for achieving maximum surface relief modulation [24]. To obtain an angular selectivity curve of a recorded grating, a vertically polarized Helium-Neon laser (He-Ne) of wavelength $633 \mathrm{~nm}$ was used as a probe beam incident at the Bragg angle to the grating (simplified drawing of the optical set-up is included in supplementary material, Fig. S1). The intensity of the 1st order diffracted beam was measured using an optical power meter to determine the diffraction efficiency of the recorded gratings. The diffraction efficiency was determined as the ratio of the intensity of diffracted light in the first diffraction order and the intensity of the incident probe beam.

After holographic recording the SRGs were exposed to UV light for $15 \mathrm{~min}$ to achieve full monomer polymerization. The process of grating formation can be explained as follows. When a photopolymer recording material is illuminated with an interference pattern of light, polymerization takes place schematically at the peaks of the structure shown in Fig. 1(b). This results in the depletion of monomer in the exposed regions and a concentration gradient of the monomer leads to the diffusion of monomer from the unexposed regions to the exposed regions. The overall refractive index is higher in the polymerized region than the unpolymerized region due to the increased density. Holograms recorded by this mechanism are known as phase holograms. The diffraction efficiency of the hologram depends on the extent of refractive index modulation, $\Delta \mathrm{n}$, which in turn depends on the rates of polymerization and diffusion within the material. It has been previously observed that in addition to the volume holographic structure, a surface relief structure with amplitude strongly depending on the spatial frequency of the recorded grating is developed. The amplitude of the surface structure can be additionally increased by exposure to elevated temperature [24]. This approach was used in the present study. In order to obtain surface relief structures with high amplitude the samples were placed in an oven (Lennox laboratory supplies Ltd) set at $120^{\circ} \mathrm{C}$ to be thermally treated. The oven temperature was increased by $20^{\circ} \mathrm{C}$ every $20 \mathrm{~min}$ until $220^{\circ} \mathrm{C}$. It was found that the gradual exposure to elevated temperature is crucial for the formation of a stable SRG. Instant exposure to elevated temperature above $160{ }^{\circ} \mathrm{C}$ leads to the complete destruction of the recorded gratings, both for volume and surface gratings. The contribution of the two gratings to the diffraction efficiency of the recorded structure was analysed before and after exposure to elevated temperature.

\subsubsection{Photonic structures coated with LTL-zeolite nanoparticles}

Following the preparation of the SRG, the layers were characterised 


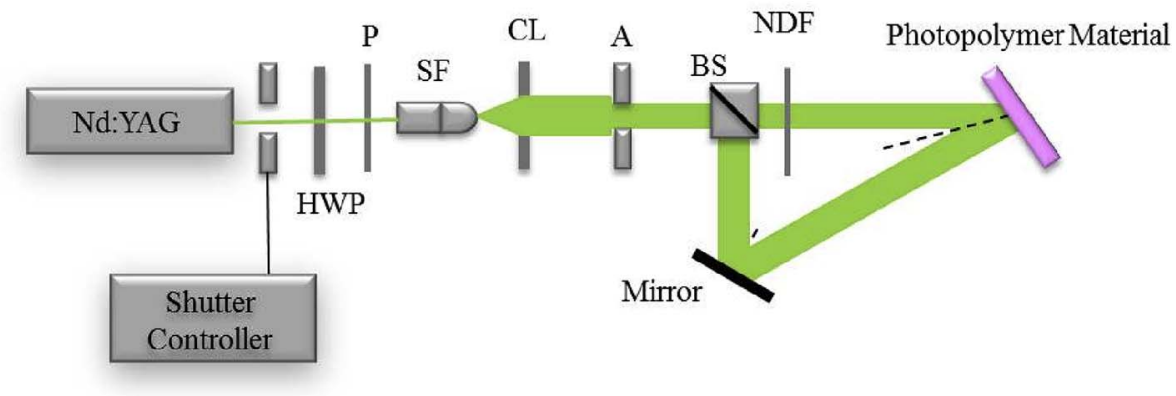

Fig. 2. Experimental set-up for recording transmission holograms: Nd:YAG: Neodymium doped yttrium aluminium garnet laser, Shutter Controller, HWP: half wave plate, P: Polariser, SF: spatial filter, CL: collimating lens, A: Aperture, BS: beam splitter, NDF: Neutral density filter.

by White Light Interferometry and by measurement of the Bragg angle before and after thermal treatment. After this, they were coated with LTL-zeolite nanoparticles incorporated in a tertaethyl ortosilicates (TEOS) layer. The layer was prepared by a sol gel process. A typical composition consisted of tetraethyl orthosilicate TEOS ( $24 \mathrm{~mL}$ ), ethanol (17.5 mL), 1.5\% wt/wt LTL-zeolite nanoparticle suspension $(12 \mathrm{~mL})$ and $0.04 \mathrm{M}$ nitric acid (3 mL) [25]. The added amount of LTL-zeolites was varied between $8 \mathrm{~mL}$ and $15 \mathrm{~mL}$ and was optimised at $12 \mathrm{~mL}$. The use of the TEOS was necessary in order to stabilise (improve mechanical stability) of the layer of nanoparticles in water environment. The porous matrix allows pollutants (in this case di-cations such as $\mathrm{Cu}$ (II) to diffuse easily into the functionalized layer and to interact with the zeolite nanoparticles. The targeted concentration detection was in the range 1-4 $\mathrm{mM}$ copper ions. The optimisation involved finding the maximum amount of zeolite nanoparticles that can be incorporated in a functionalising layer while still preserving a good optical quality. The solution was stirred for $24 \mathrm{~h}$ and then spin coated at $500 \mathrm{rpm}$ on the thermally treated SRG structures.

The samples were left for $24 \mathrm{~h}$ at room temperature before further studies. Next the gratings were exposed to the different copper solution for pre-determined time intervals and their diffraction efficiency was measured following the flow chart shown in Fig. S3. The layers were studied under white light interferometer and the diffraction efficiency change was measured at different stages.

\section{Results and discussions}

\subsection{Characterisation of the LTL zeolite nanoparticles and the TEOS/LTL composite layer}

The size of the nanoparticles was confirmed to be $80 \mathrm{~nm}$ as aggregates and was measured by dynamic light scattering (DLS) (Fig. 3(a)), while the individual size of the zeolite grains was in the range $10-20 \mathrm{~nm}$ (Fig. S2(a)) according to the high-resolution transmission electron microscopy study (HRTEM). It can be clearly observed, that the LTL sample is fully crystalline (Fig. 3(b)) and consists of many aggregates, which are formed from single rectangular crystalline domains with well-defined edges and crystalline fringes Fig. S2 (b) reported in supplementary material. The porosity of the TEOS layer was confirmed by the SEM images example of which is presented in Fig. S2 (c). It was also observed that the LTL nanoparticles are homogeneously dispersed in the TEOS layer as seen in the SEM study of the TEOS/LTL nanocomposite (Fig. S2 (d)).

\subsection{Characterisation of holographic surface relief photonic structures}

Profiles of the surface relief structure measured by white light interferometer are presented in Fig. 4. The spatial frequency of the structure was determined to be 345 lines/mm from the $3.1 \mu \mathrm{m} \pm$ $0.2 \mu \mathrm{m}$ line spacing observed in the white light interferometry (WLI) results. Previous studies revealed that peaks and troughs of the surface relief structure correspond respectively to the bright and dark regions in the pattern confirming that mass transport from dark to bright regions is the main mechanism of photoinduced surface relief formation [24]. The amplitude of the troughs observed by these images was in the range $350 \mathrm{~nm}-400 \mathrm{~nm}$ Fig. 4(a). Fig. 4 (a) and (b) shows 3D surface topography of the grating after thermal treatment, and after spin coating with the modified LTL-zeolite layer. After spin coating with the LTLzeolite layer, the surface relief amplitude has decreased from $350 \mathrm{~nm}$ to approximately $20 \mathrm{~nm}$ indicating that the TEOS/LTL nanocomposite has filled in the troughs (Fig. 4(b)). The diffraction efficiency and the surface profile of the SRG structures were measured at each stage of the experiment.

\subsection{Diffraction efficiency and angular selectivity studies}

Fig. 5 shows the diffraction efficiency as a function of angle of rotation for the photonic gratings at different stages of the experiment i.e. before thermal treatment, after thermal treatment, after coating with LTL/TEOS layer, and after exposure to copper ions. A maximum diffraction efficiency of the photopolymer layers of $60 \%$ was achieved immediately after holographic recording and as outlined in Table 2. This value is due to the volume diffraction grating since at this stage the surface relief grating has negligible amplitude $(<1 \mathrm{~nm})$. After thermal treatment the diffraction efficiency decreased to ${ }^{\circ} 35 \%$ due to combined effects of enhancement of the surface relief grating and nearly complete erasure of the volume grating. This was confirmed by experiment in which the post thermally treated grating was coated with an index matching liquid. The diffraction efficiency of the volume grating after index matching was measured to be $2.5 \%$, therefore, after thermal treatment the diffraction efficiency is mainly due to the SRG. A shift in the Bragg angle was observed as it can be seen in Fig. 5. The shift is most probably due to an increase in the effective refractive index of the photopolymer layer, as the density of the layer is increased after thermal treatment. Taking into account the height of the surface relief amplitude - $400 \mathrm{~nm}$ and the diffraction efficiency of the structure, the refractive index modulation is estimated to be 0.9 . Other possible causes of the shift are shrinkage of the layer and a change of the spatial frequency of the structure. The Bragg angle measurement was then taken for layers modified with zeolites nanoparticles dispersed in TEOS. A further decrease in the diffraction efficiency was seen at this point due to substitution of the air in the troughs with material with higher refractive index. Assuming that the LTL/TEOS nanocomposite fully covers the surface relief structure, based on the measured diffraction efficiency after coating (20\%) one can estimate the refractive index of the coating layer to be 1.4 , which is a reasonable value taking into account the porosity of the material. Further decrease of the diffraction efficiency was observed under exposure of the sample to the analyte. Spectrophotometric studies of the absorption due to presence of Copper (II) in water solution revealed that the absorption losses were varying between 0.9 and $3.6 \%$ when the copper concentration was varied between 1 and $4 \mathrm{mM}$. This corresponds to an estimated maximum variation of the absolute diffraction efficiency (which at the start of the experiment is $20 \%$ ) from $19.82 \%$ for $1 \mathrm{mM}$ solution to $19.28 \%$ for the 
a)

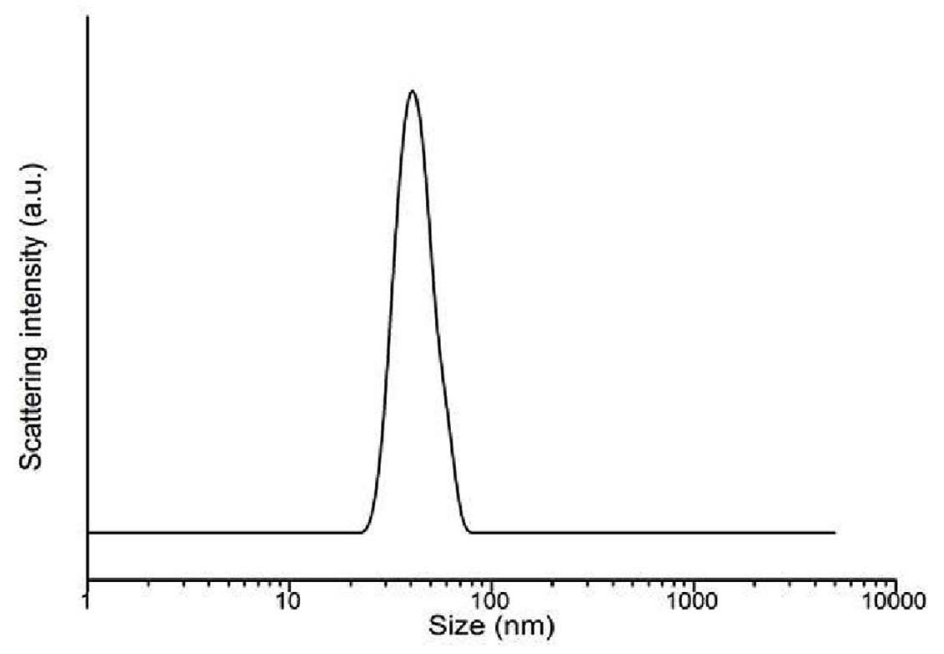

b)

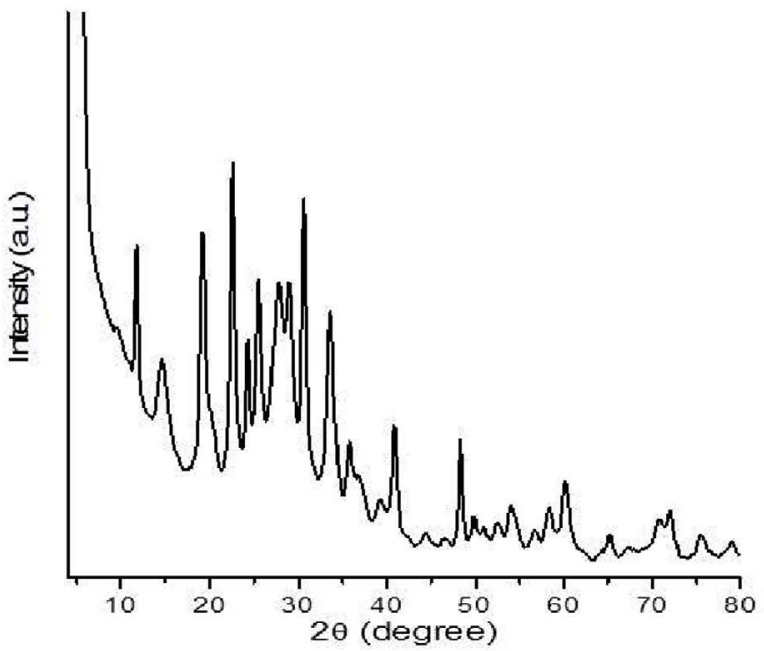

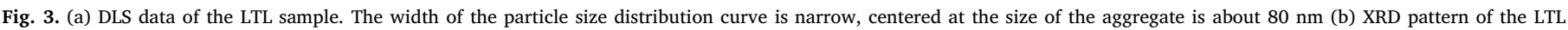
nanoparticles.

$4 \mathrm{mM}$ solution. These variations were much smaller than the decrease caused by the change in the refractive index. Detailed dependence of this decrease on analyte concentration is presented in section 4.4.

\subsection{Exposure to the target analyte}

In order to determine the applicability of the proposed holographic sensor for detection of analytes, the zeolite-coated SRG was exposed to copper solutions with 1-4 mM concentration following a procedure described in the flowchart presented in supplementary data Fig. S3. All the molar concentrations were prepared in deionised water.

In order to check whether the sensor response is due to the TEOS or due to the presence of LTL-zeolites nanoparticles, the SRG grating was also coated with TEOS containing no nanoparticles as a reference. Fig. 6(a) shows the diffraction efficiency versus exposure time for SRG coated with TEOS only and TEOS containing zeolites to a $4 \mathrm{mM}$ Copper solution. A significant improvement in the sensor response to copper is observed due to the inclusion of LTL zeolites. In Fig. 6(b), the normalised diffraction efficiency vs exposure time is shown for the LTL zeolite coated SRG exposed to fresh water and 1-4 mM copper solutions. The normalised diffraction efficiency was observed to decrease in

a)

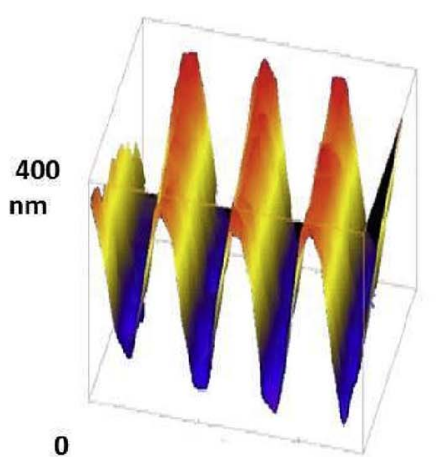

b)

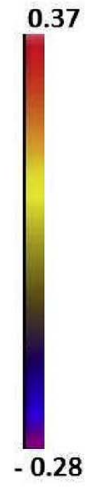

all cases with increasing exposure time and a clear dependence on the concentration of copper was observed. However, it was also observed that on exposure to the control solution, deionised water, the normalised DE drops down from 1 to 0.8 .

The selectivity of the proposed sensor is determined by the selectivity of the LTL nanoparticles. It was observed that much smaller changes in the diffraction efficiency were detected for monovalent cations than for divalent cations. For example after $3 \mathrm{~min}$ of exposure to a solution containing $4 \mathrm{mM}$ of $\mathrm{Na}^{+}$, a $13 \%$ decrease in diffraction efficiency was measured. Exposure for $3 \mathrm{~min}$ to $4 \mathrm{mM}$ solutions of $\mathrm{Cu}^{2+}$ and $\mathrm{Ca}^{2+}$ led to correspondingly $60 \%$ and $44 \%$ decrease in diffraction efficiency. All changes in diffraction efficiency were calculated with respect to the reference values of diffraction efficiency of devices exposed to water in order to ensure that only the change due to the presence of the specific analyte is taken into account. At shorter exposure time, $2 \mathrm{~min}$, no change in diffraction efficiency due to the presence of monovalent $\mathrm{Na}^{+}$cations was measured, while the changes in diffraction efficiency for $\mathrm{Cu}^{2+}$ and $\mathrm{Ca}^{2+}$ were correspondingly $51 \%$ and $25 \%$.
Fig. 4. WLI images (3 dimensional views) of the SRG of spatial frequency 300 lines/mm (a) SRG and (b) SRG with LTL-zeolites nanoparticles. 


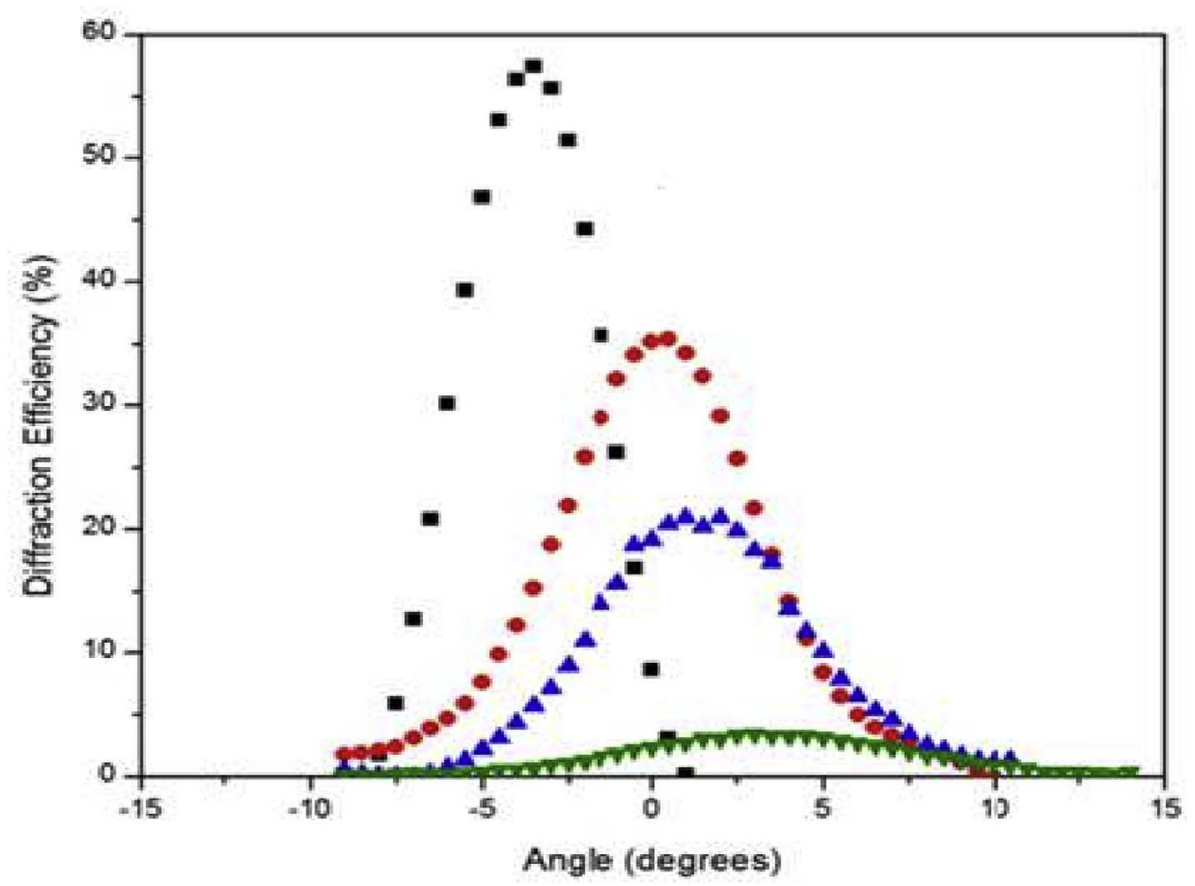

Fig. 5. Bragg curves with spatial frequency centered at $300 \mathrm{l} / \mathrm{mm}$ recorded in layers with thickness of $30 \mu \mathrm{m}$ on a glass slide taken for the photopolymer before and after thermal treatment $\mathrm{C}$, after spin coating with LTL-zeolites $\nabla$, and after exposure to Copper (II) analyte $\nabla$ Flow chart of this experiment can be found in Fig. S3.

Table 2

Diffraction efficiency and amplitude of SRG at different stages of the experiment.

\begin{tabular}{lll}
\hline Photonic structure & $\begin{array}{l}\text { Diffraction } \\
\text { Efficiency (DE \%) }\end{array}$ & $\begin{array}{l}\text { Surface modulation } \\
\text { (nm) }\end{array}$ \\
\hline $\begin{array}{c}\text { Photopolymer layer (volume } \\
\text { grating) }\end{array}$ & $60 \% \pm 5$ & $<1$ \\
$\begin{array}{c}\text { Post thermal treatment (surface } \\
\text { grating) }\end{array}$ & $35 \% \pm 3$ & 400 \\
$\begin{array}{c}\text { TEOS/zeolite coated grating } \\
\text { (modified surface grating) }\end{array}$ & $20 \% \pm 2$ & 20 \\
$\begin{array}{c}\text { TEOS/zeolite coated grating } \\
\text { exposed to Cu }{ }^{+2} \text { for } 3 \text { min }\end{array}$ & $3 \% \pm 1$ & 30 \\
\hline
\end{tabular}

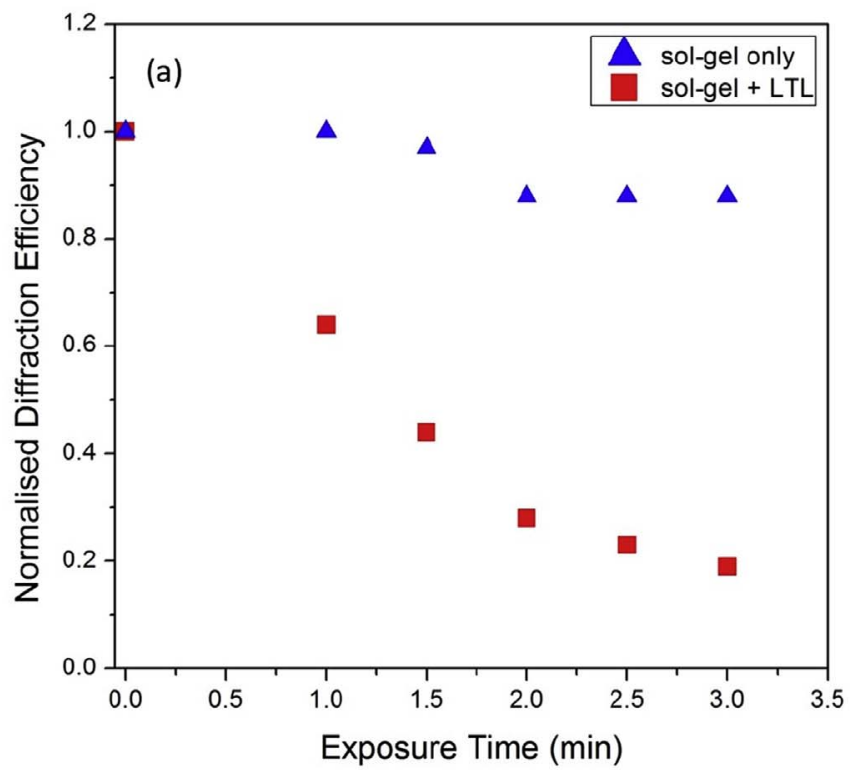

\subsection{Theoretical modeling of the results}

Fig. 7 shows the theoretically modelled diffraction efficiency in the first order of diffraction ( $m=1$ ) of a surface relief grating with amplitude of $400 \mathrm{~nm}$ as a function of grating change in refractive index $\Delta n$. A value of reconstruction wavelength of $633 \mathrm{~nm}$ was used for this calculation. Due to the oscillatory nature of the Bessel function in eqn. (2), the diffraction efficiency of the grating is observed to either increase or decrease with increasing $\Delta n$ corresponding to the left or right hand side of theoretical curve. Therefore, the sensor output may register the decrease in grating $\Delta n$ associated with ion exchange of analyte molecules as either an increase or decrease in grating diffraction efficiency, depending on which side of the curve the sensor is operating in. It is assumed in this work that on ion exchange of $\mathrm{Cu}^{2+}$ ions to the LTL zeolite nanoparticles, the overall value of $\Delta n$ for the SRG will decrease.

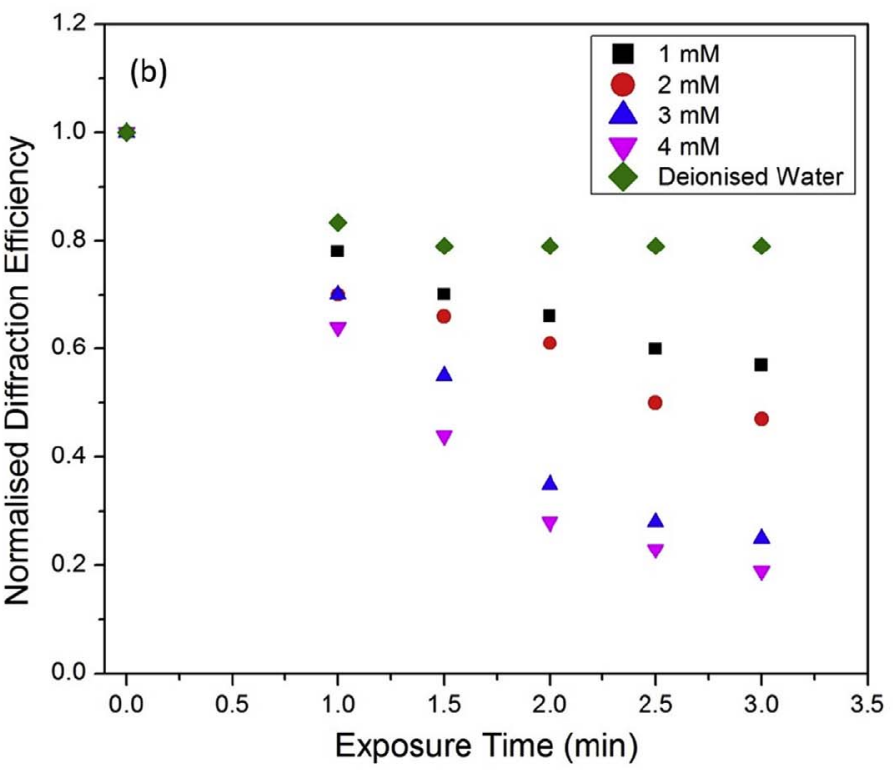

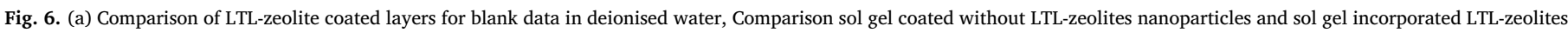
nanoparticles in $4 \mathrm{mM} \mathrm{Cu}$ (II) solution (b) Copper exposure (1-4 mM) response in terms of diffraction efficiency change of LTL-zeolites nanoparticles photonic structures. 


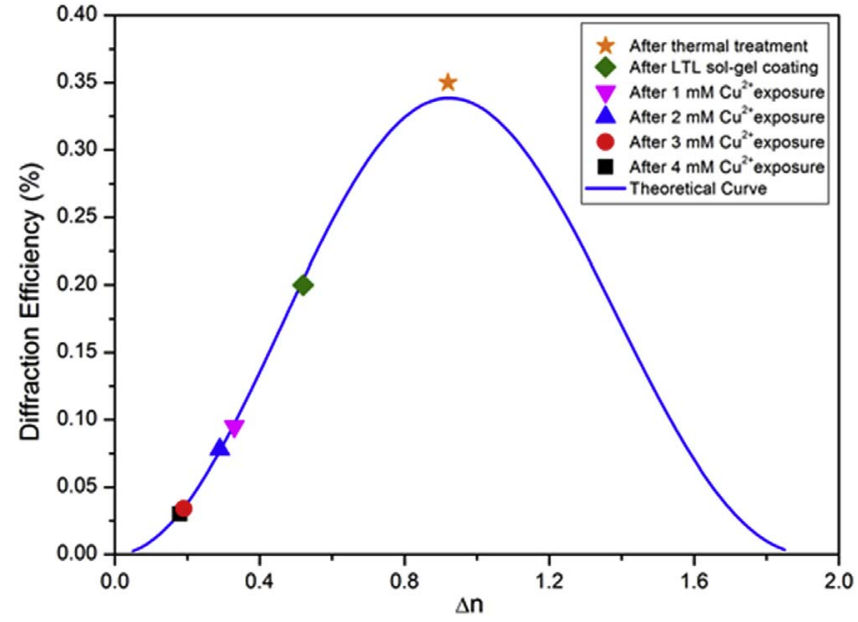

Fig. 7. Diffraction Efficiency vs. change in refractive index $\Delta$ n. Blue line $=$ theoretical data, coloured points $=$ experimental data. (For interpretation of the references to colour in this figure legend, the reader is referred to the web version of this article.)

Therefore, for this theoretical study we expect that the sensor is operating in a regime based on the left hand side of the theoretical Bessel curve. However, further experimental measurements are underway to verify this.

The blue line in Fig. 7 represents the theoretical data and the coloured points correspond to the experimentally measured diffraction efficiency values for a $400 \mathrm{~nm}$ surface relief grating initially uncoated (35\%), after coating with LTL zeolite nanoparticles (20\%), and after 3 min exposure to a $\mathrm{Cu}^{2+}$ solution at concentrations of $1-4 \mathrm{mM}$. For concentrations of 1 and $2 \mathrm{mM} \mathrm{Cu}^{2+}$, reductions in $\Delta n$ to 0.195 and 0.230 are required to produce the experimentally observed $10.5 \%$ and $12.2 \%$ decreases in diffraction efficiency due to the presence of copper respectively. There is less clear distinction between the 3 and $4 \mathrm{mM}$ concentrations: reductions in $\Delta n$ of 0.030 and 0.034 are required to produce the experimentally observed decreases in SRG diffraction efficiency of $18 \%$ and $18.6 \%$, respectively. The difficulty in distinguishing the 3 and $4 \mathrm{mM}$ concentrations may be due to the fact that the $\Delta n$ values after copper adsorption are located at the curved base of the Bessel function where the slope is greatly reduced in comparison to the linear portion; therefore, a greater change in $\Delta n$ will be required to produce a measurable change in diffraction efficiency than would be required for $\Delta n$ values located along the linear section of the Bessel function. The location of the initial $\Delta n$ along the Bessel function should thus be carefully considered when designing a thin SRG-based sensor [26].

\section{Conclusions}

A method of fabrication of a water pollutant sensor by holographic recording of surface relief structures in a self-processing photopolymer material and modified by spin coating LTL-zeolites in a sol-gel matrix is presented. The sensor was confirmed to respond to copper ions at concentration of 1-4 mM with good reproducibility. The proposed technology platform for preparation of sensors is flexible, because the selectivity can be easily changed by modification of the functionalising layer chemical structure. The sensor also demonstrates good linearity limit for $\mathrm{Cu}^{2+}$. The loadings of LTL-zeolites were tailored in order to detect $63 \mathrm{ppm}$ and successful to determine $\mathrm{Cu}^{2+}$ in real samples. To the best of the author's knowledge, this is the first use of SRG coated with a sensing layer for copper ion detection. The disposable holographic sensor also has a relatively quick response time, is low cost, and allows for real time monitoring of environmental water quality.

\section{Acknowledgements}

This work was supported by DIT Fiosraigh Scholarship. The authors express their cordial gratitude to Focas Research Institute at DIT for providing access to their experimental facilities. The authors would like to acknowledge the contribution of Dr. Luke O'Neill and Dr. Brian Goery who collected the SEM data for TEOS and TEOS/LTL nanocomposite. The authors are also thankful to the IEO team members for their co-operation during the execution of this research.

\section{Appendix A. Supplementary data}

Supplementary data related to this article can be found at http://dx. doi.org/10.1016/j.micromeso.2017.11.019.

\section{References}

[1] F. Fu, Q. Wang, J. Environ. Manage 92 (2011) 407-418.

[2] E. Swaminathan, S. Nagappan, Padmavathi Rajangam, Sangeetha Dharmalingam, Prog. Nanotechnol. Nanomater 2 (2013) 47-54.

[3] C.K. Ho, A. Robinson, D.R. Miller, M.J. Davis, Sensors 5 (2005) 4-37.

[4] A.K. Yetisen, I. Naydenova, F. Da Cruz Vasconcellos, J. Blyth, C.R. Lowe, Chem. Rev. 114 (2014) 10654-10696.

[5] S.C. Byun, Y.J. Jeong, J.W. Park, S.D. Kim, H.Y. Ha, W.J. Kim, Solid State Ionics 177 (2006) 3233-3243.

[6] M. Zawadzka, T. Mikulchyk, D. Cody, S. Martin, A.K. Yetisen, H.B. Juan Leonardo Martinez-Hurtado, E. Mihaylova, H. Awala, S. Mintova, S.H. Yun, I. Naydenova, Photonic materials for sensing, Biosensing Disp. Devices (2016) 315-359.

[7] M. Hölzl, S. Mintova, T. Bein, Stud. Surf. Sci. Catal. 158 (2005) 11-18.

[8] S. Mitchell, A.B. Pinar, J. Kenvin, P. Crivelli, J. Perez-Ramirez, Nat. Commun. 6 (2015) 8633.

[9] D. Mao, Y. Geng, H. Liu, K. Zhou, L. Xian, D. Yu, Appl. Opt. 55 (2016) 6212-6221.

[10] G. Ye, X. Li, X. Wang, ChemComm 46 (2010) 3872-3874.

[11] V.K.S. Hsiao, J.R. Waldeisen, Y. Zheng, P.F. Lloyd, J. Bunning, T.J. Huang, J. Mater Chem. 17 (2007) 4896-4901.

[12] X. Wang, X. Wang, Chem. Commun. 49 (2013) 5957-5959.

[13] Z. Zhou, Z. Shi, X. Cai, S. Zhang, S. Corder, X. Li, Y. Zhang, G. Zhang, L. Chen, M. Liu, D. Kaplan, F. Omenetto, Y. Mao, Z. Tao, T.H. Tao, Adv. Mater 29 (2017) 1605471.

[14] W. Chien, M. Zeeshan, X. Dai, A.G. Kirk, 138 (2009) 441-445.

[15] T.L. Lowder, J.D. Gordon, S.M. Schultz, R.H. Selfridge, Opt. Lett. 32 (2007) 2523-2525.

[16] T.L. Lowder, K.H. Smith, B.L. Ipson, A.R. Hawkins, R.H. Selfridge, S.M. Schultz, IEEE Photonics Technol. Lett. 17 (2005) 1926-1928.

[17] Y. Fuchs, S. Kunath, O. Soppera, K. Haupt, A.G. Mayes, Adv. Funct. Mater 24 (2014) 688-694.

[18] J.J. Cowan, J. Opt. Soc. Am. A 7 (1990) 1529.

[19] A.K. Yetisen, H. Butt, T. Mikulchyk, R. Ahmed, Y. Montelongo, M. Humar, N. Jiang, S. Martin, I. Naydenova, S.H. Yun, Adv. Opt. Mater 4 (2016) 1589-1600.

[20] W.R. Klein, B.D. Cook, IEEE Trans. Sonics Ultrason. 14 (1967) 123-134.

[21] H. Kogelnik, Bell Syst. Tech. J. 48 (1969) 2909-2947.

[22] C.V. Raman, N.S.N. Nath, Proc. Indian Acad. Sci. - Sect. A 84 (1936).

[23] A. Kharchenko, O.I. Lebedev, V. Zholobenko, V. de Waele, S. Mintova, J. Phys. Chem. C 120 (2016) 26300-26308.

[24] K. Trainer, K. Wearen, D. Nazarova, I. Naydenova, V. Toal, J. Opt. 12 (2010) 124012.

[25] S. Jaiswal, P. McHale, B. Duffy, Colloids Surfaces B Biointerfaces 94 (2012) $170-176$.

[26] D. Cody, I. Naydenova, Theoretical modelling and design of photonic structures in zeolite nanocomposites for gas sensing: part I - surface relief gratings, JOSA A 34 (2017) 2110-2119. 